\title{
Digestive Diseases
}

Adamek, H.E. 18

Armellini, E. 63

Bassler, B. 18

Chou, P. 87

Corazza, G.R. 63

Di Sabatino, A. 63

Ebert, M. 92

Gerstenhauer, G. 81

Gritzmann, N. 6

Hartmann, D. 18

Hollerweger, A. 6

Hori, M. 39

Hübner, E. 6

Kahl, S. 26
Kim, T. 39

Layer, G. 18

Lee, W.-L. 87

Liu, C.-M. 87

Liu, J.-H. 87

Lochs, H. 67

Macheiner, P. 6

Malfertheiner, P. 5, 26, 32, 92

Mathis, G. 73

Murakami, T. 39

Nakamura, H. 39

Neye, H. 67, 81

Ocran, K.W. 81

Peschl, R. 73
Rickes, S. 5, 32, 67, 81, 92

Riemann, J.F. 18

Schilling, D. 18

Schütte, K. 92

Singh, A.K. 56

Tomoda, K. 39

Tung, T.-H. 87

Voderholzer, W. 67

Weber, J. 67

Werle, A. 73

Wermke, W. 67, 81

Zalis, M. 56

\section{Subject Index Vol. 22, No. 1, 2004}

Adenocarcinoma 26

Adenoma 26

Community-based study 87

Computed tomography 6

Contrast agent 73

Crohn's disease 63, 67

Cross-sectional imaging 56

CT 56

Diagnostic criteria, evaluation 67

Differential diagnosis 32,81

Digestive system neoplasms 26

Disease activity 67

Doppler sonography 63

Ductal cell adenocarcinoma 6

Echo-enhanced sonography 32,81

Endoscopic retrograde cholangiopancreatography 18

Endosonography 26

Epithelial cystic neoplasms 18

EUS, review of the literature 26

Focal liver lesion 73

- nodular hyperplasia 73
Functional tumors of the pancreas 6 Gallstone disease, prevalence 87

Haemangioma 73

Hepatocellular carcinoma 73

Ileocolonoscopy 67

Inflammatory bowel disease 56

Insulinoma 6

Intramural vascularity 63

Islet cell tumors 18

- $\quad$ - , diagnosis 26

Levovist 63

Liver, diseases 39

- metastases 81

- neoplasms, CT 39

- -, diagnosis 39

- - , MR 39

Magnetic resonance cholangiopancreatography 18

- - imaging 18

Metastasis 73

MRI 56

Mucinous tumors 6
Neoplasm staging 26

Pancreas tumors 6

Pancreatic cancer 18

- neoplasms 26

- tumors 6, 32

Pancreatitis 26

Power Doppler sonography 67

Prospective study 67

Serous cystadenoma 6

Solid pancreatic tumors, surgery 26

Staging 6

Type 2 diabetes 87

Ulcerative colitis 63

Ultrasonography, interventional 26

Ultrasound 73

- signal enhancer 73

\section{KARGER}

(C) 2004 S. Karger AG, Basel

Fax +4161306 1234

E-Mail karger@karger.ch

www.karger.com 